\title{
La economía política de la escala y la construcción de las regiones transfronterizas*
}

\begin{abstract}
The construction of crossbor der regions is related to economic, political and social rescaling general processes; they both respond and contribute to the "scale relativization" associated with the fall, since '80s, of the relative coherence structured between national economy, State and society that had characterized postwar boom peak. This is reflected in a proliferation of scales on which attempts of restructuring economic, political and social relationships occur. This paper explores some of this processes and strategies linked to the emergency and consolidation of crossborder regions, and comments about their meaning for economic and political restructuration.
\end{abstract}

Keywords: restructuration, rescaling, crossborder regions.

\section{Resumen}

La construcción de las regiones transfronterizas (RTF) se r elaciona con los procesos generales de reescalamiento económico, político y social; ambos responden y contribuyen a la "relativización de la escala" asociada con el declive, desde inicios de los '80, de la relativa coherencia estructurada entre la economía, el Estado y la sociedad nacional que había caracterizado el apogeo del boom de la postguerra. Esto se refleja en una proliferación de escalas sobre las cuales ocurren intentos de reestructurar las relaciones económicas, políticas y sociales. Este artículo explora alguno de estos procesos y estrategias vinculadas a la emergencia y consolidación de RTF, y comenta sobre su significación para la reestructuración económica y política.

Palabras clave: reestructuración, reescalamiento, regiones transfronterizas. 


\section{Introducción}

$\mathrm{L}$ a construcción de las regiones transfronterizas, como un ejemplo de microrregionalismo, se relaciona de mejor forma con los procesos generales de reescalamiento económico, político y social ${ }^{1}$. Pues ambos responden y contribuy en a la "relativización de la escala" asociada con el declive, desde inicios de los ' 80 , de la relativa coher encia estructurada entre la economía nacional, el Estado nacional y la sociedad nacional que había caracterizado el apogeo del boom de postguerra. La crisis del modelo de crecimiento económico de postguerra en las economías capitalistas avanzadas, el fin de la Segunda Guerra Fría, el surgimiento del neoliberalismo global, el quiebre del bloque soviético, la apertura de China al capital externo y el creciente número de los llamados "estados fallidos" han contribuido a esta relativización de la escala. Esto se refleja en una proliferación de escalas sobre las cuales ocurren intentos de reestructurar las relaciones económicas, políticas y sociales -que van desde políticas para promover la globalización económica, la gobernanza global y la cultura global a través de varias formas de mega, macro y mesorregionalismo, hasta preocupaciones sobre las economías microrregionales, el empoderamiento de comunidades locales e identidades "tribales". Este artículo explora alguno de estos procesos y estrategias vinculadas a la emergencia y consolidación de regiones transfronterizas y comenta sobre su significación para la reestructuración económica y política.

\section{La primacía de la escala nacional en el capitalismo de posguerra}

En los "treinta años gloriosos" de la expansión económica de postguerra, la escala nacional dominó la organización económica. De esta forma,

\footnotetext{
Sociólogo, director del Instituto de Estudios Avanzados en G estión y Ciencias Sociales, profesor del Departamento de Sociología de la Universidad de Lancaster (Inglaterra). E-mail: r.jessop@lancaster.ac.uk

** Traducido por Luis Riffo Pérez.

${ }^{1}$ Este artículo se ha beneficiado de los comentarios de Neil Brenner, Martin Jones, Markus Perkmann, Ngai-Ling Sum y Fredrik Söderbaum.
}

el complejo campo de las relaciones económicas fue manejado como si estuviera dividido en un conjunto de economías nacionales relativamente cerradas. La primacía de la economía nacional y el Estado nacional en el fordismo atlántico estuvo vinculada al cierre relativo de las economías de postguerra, las que estaban experimentando una reconstrucción más o menos significativa sobre la base de la producción y el consumo masivo. Mientras que las instituciones y regímenes internacionales fueron organizados para rescatar a los estado nacionales europeos y asegurar el crecimiento económico nacional, sus estados subnacionales (regionales o locales) actuaron fundamentalmente como mecanismos de transmisión de la política nacional. En varios de los regímenes mercantilistas del Este asiático o "naciones comerciales", ocurrió el mismo tipo de "naturalización" a través de la prominencia del discurso de la "seguridad nacional”, que vinculó la seguridad interna o externa a un fuerte control sobre la economía doméstica. La industrialización por substitución de importaciones tuvo efectos similares en muchas economías latinoamericanas. La continua internacionalización económica de estos diferentes tipos de economías y las crisis emergentes de sus respectivos modelos de crecimiento, sirvieron para debilitar la noción aceptada de la economía nacional como objeto de gestión económica.

También se vio crecientemente que las economías regionales y locales tenían sus propios problemas específicos, los cuales no podían ser resueltos por medio de políticas macroeconómicas nacionales ni por políticas meso o macroeconómicas impuestas desde arriba por los estados nacionales. Reemplazando a la economía nacional como el objeto primario (y objetivo) de la gobernanza económica para la mayoría de los estados miembros de la OCDE, está la economía basada en el conocimiento (EBC), en una era de globalización. La EBC se anuncia como la última etapa del capitalismo y su expansión está siendo promovida para superar las crisis económicas de los ' 80 y ‘ 90 . Ella se vincula con narrativas de competitividad internacional, flexibilidad, capital social, aprendizaje, confianza, reflexividad y gobernanza descentralizada, así como con el creciente rol de las nuevas tecnologías de la información y comunicación, 
productos "inteligentes" y servicios exper tos. La promoción de la EBC también se vincula con nuevas formas de coordinación y/o regulación económica supranacional, así como con llamados a una mayor autonomía regional y local para seguir estrategias de ofer ta adecuadas en sus puntos de aplicación. Estas reorientaciones estratégicas intensifican el desarrollo desigual, generando sin embargo intentos de contrarrestar sus efectos en las regiones más marginalizadas, periféricas o no competitivas. Variaciones en estas estrategias y contraestrategias se reflejan en las cambiantes estrategias económicas seguidas en diferentes RTF.

El alcance para el regionalismo a diferentes escalas también se expandió con el fin de la Segunda Guerra Fría. Junto a los nuevos tipos de vínculos meso y macrorregionales asociados con nuevas formas de rivalidad en Europa y el mundo, especialmente por la influencia en las economías postsocialistas, existen muchas oportunidades para articular regiones fronterizas previamente periféricas. Esto se aplica no sólo a los nuevos estados nacionales del ex bloque soviético, sino también a otras sociedades postsocialistas o en transformación. El crecimiento de las RTF en las fronteras de las economías postsocialistas y/o entre economías postsocialistas y sus vecinos capitalistas son dos expresiones importantes de este fenómeno. El fin del sistema mundial bipolar ha transformado, más que terminado, las cuestiones sobre seguridad, y ellas aún influyen en las perspectivas para la cooperación en Europa. Problemas similares pueden encontrarse en el desarrollo económico en estrechos como el de Taiwán y la República Popular China; el Área de Desarrollo del Río Tumen, que incluye partes de Rusia, China, Mongolia, Japón, Corea del Norte y Corea del Sur (Jordan \& Khanna, 1995); la Sub-región del Gran Mekong; y áreas de guerra civil o conflictos internacionales en el Cuerno del África y el África Subsahariana o en los Balcanes. Los temas de seguridad e inmigración son también fuentes de fricción en el desarrollo de la cooperación transfronteriza en la frontera de Estados Unidos y México (Scott, 2002).

\section{La relativización de la escala}

Aunque estos procesos han degradado la escala nacional tal como estaba constituida en el periodo de postguerra como la base para organizar las relaciones económicas, políticas y sociales, ninguna otra escala de organización económica y política (ya sea local, global, urbana, triádica, regional o suprarregional) ha ganado aún una primacía similar. Collinge (1996) se refiere a este fenómeno como la relativización de la escala.

En efecto, diferentes espacios económicos y políticos están compitiendo para convertirse en el nuevo ancla alrededor de la cual otras escalas (sin impor tar cuántas y cuáles) puedan organizarse para producir un adecuado grado de coherencia estructurada. Esto implica proyectos económicos y políticos orientados a escalas diferentes, y aún no se produce consenso sobre como éstos serán reconciliados -lo que se refleja en continuos debates y en activas respuestas sobre la importancia relativa de sitios y espacios globales, nacionales y regionales de acción económica. En este contexto, es importante notar que no es que haya un conjunto predeterminado de lugares, espacios o escalas que estén simplemente siendo reordenados. Pues los lugares, espacios y escalas no son predeterminados, sino que son sujetos a luchas discursivas sobre mapeo y denominación (Neumann, 1993; Jenson, 1995; Paasi, 2001) y a luchas más sustantivas sobre su institucionalización social, material y espacio-temporal. De esta forma, encontramos que nuevos lugares están emergiendo, nuev os espacios están siendo creados, nuevas escalas de organización están siendo desarrolladas y nuevos horizontes de acción están siendo imaginados. En particular, lo global es sólo una de las muchas escalas sobre las cuales se están imaginando y siguiendo intentos de reestabilizar el capitalismo. En efecto, la globalización a menudo se vincula estr echamente, y en formas complejas, con procesos ocurridos en otras escalas espaciales. Se puede concebir mejor como parte de una proliferación de escalas y temporalidades narradas como objetos de acción institucionalizados, regularización y gobernanza. 
De esta forma, mientras que ha habido un creciente énfasis sobre cómo organizar mejor la escala global alineada con un amplio rango de criterios a menudo conflictivos, las pequeñas escalas (notablemente lo urbano, regional y nacional) aún son significativas (aunque a menudo bajo nuevas formas) como sitios sustantivos de actividades económicas reales. Muchas estrategias también están siendo desarrolladas para vincular éstas y otras escalas a lo global -incluyendo la internacionalización, triadización, formación de bloques regionales, construcción de redes de ciudades globales, formación de regiones transfronterizas, localización internacional, glocalización, glurbanización y transnacionalización ${ }^{2}$. La emergencia del ciberespacio como una arena de acción vir tual, que parece que estuviera en todas partes y en ninguna a la vez, ha complicado más aún estos problemas. Más aun, a medida que nuevas escalas emergen y/o las escalas existentes ganan en densidad institucional, las fuerzas sociales también intentan desarrollar nuevos mecanismos que las articulen o coordinen. Esto añade niveles adicionales de complejidad y puede gatillar nuevos combates de reacción y contrarreacción, a medida que los eventos o procesos ocurridos en una escala se usan para justificar acciones en otras escalas. Por ejemplo, la internacionalización a menudo provoca respuestas regionales (Perkmann, 2000); la europeización requiere de la coordinación de políticas regionales (Leresche \& Saez, 2002); el desarrollo del NAFTA ha promovido la formación de la región de Cascadia abarcando desde Alaska a Oregón y el norte de California (Blatter, 2001; Sparkes, 2002). De esta forma, el resurgir de las provincias y regiones dentro de los estados territoriales, y el crecimiento de las RTF en sus franjas, son respuestas parciales a la centralización política. Estos procesos también pueden ser relacionados con preocupaciones vinculadas a la ecología política, tales como la sustentabilidad, y en algunos casos, con el declive de antiguas preocupaciones sobre seguridad y el surgimiento de nuevos problemas en los '90.

Lo anterior genera una complejidad creciente a medida que diferentes escalas de acción se rela-

${ }^{2}$ Sobre la glocalización, ver Brenner (1998) y Swyngedouw (1997); sobre glurbanización, Jessop y Sum (2000); sobre transnacionalización, Smith (2000). cionan con varias combinaciones de tipo vertical, horizontal, diagonal, centrípetas, centrífugas y vorticales. Esta complejidad no puede ser capturada en términos de simples contrastes, tales como lo global-nacional o global-local, o con conceptos híbridos tales como "glocalización" o "transversal". En lugar de esto, ahora vemos una proliferación de escalas discursivamente constituidas, institucionalmente materializadas y espacialmente incorporadas (ya sean terrestres, territoriales o telemáticas), que se relacionan en jerarquías crecientemente complejas y enredadas con diversas temporalidades y espacialidades, en vez de jerarquizarse en formas simples unas con otras.

La proliferación de horizontes espaciales y temporales vinculados con la relativización de las escalas implica oportunidades y amenazas muy diferentes para las fuerzas económicas, políticas y sociales de aquellas que prevalecieron cuando la escala nacional era juzgada como la predominante. Esto promueve acciones para explotar nuevas oportunidades de manera de promover valores, identidades e intereses específicos y/o defenderlos del impacto frecuentemente disruptivo del reescalamiento. Muchos actores y fuerzas sociales diferentes están implicados en esta explotación y disputa, desde los migrantes económicos hasta empresas legales e ilegales, estados y movimientos no gubernamentales. Los actores económicos y políticos a menudo son activos en términos de intentar fortalecer la competitividad basada en el lugar y/o fortalecer la competitividad promoviendo la movilidad. No existe una correspondencia simple entre estrategias y actores: algunas firmas tienen un vínculo territorial, otras se mueven en el espacio de los flujos; los estados pueden buscar mantener las actividades económicas en sus lugares o promover su movimiento en favor de firmas domésticas móviles. Con todo, tales actividades reordenan -a través del espacio económico en diferentes escalas espaciales- las complementariedades y diferencias basadas en lugares como la base para las ventajas competitivas dinámicas. No obstante, el juego competitivo siempre produce comparativamente perdedores, así como ganadores.

Algunos comentaristas explican las regiones o escalas emergentes como basadas en "territorios económicos naturales", los que han podido 
reemerger o desarrollarse a medida que el Estado nación declina como fuente de poder económico y político.

Ciertamente, es interesante reflexionar aquí sobre el resurgimiento de antiguos bloques comerciales transfronterizos en la era del postsocialismo y del fin de la Guerra Fría. Esto puede ser visto tanto en Europa del Este y Central así como en la "Gran China". Pero creo que los "territorios económicos naturales” (TEN) son espacios económicos que han sido "naturalizados" discursivamente, así como constr uidos económica y políticamente. Por ejemplo, el que uno vea a los TEN como naturales o no depende del entendimiento que se tenga sobre los modos dominantes de competencia y los factores causantes de la competitividad estructural. Una interpretación ricardiana (basada en un crecimiento conducido por los factores) conducirá a identificar a los TEN y a las estrategias económicas de forma diferente que aquellas interpr etaciones de tipo schumpeteriano (basadas en un crecimiento conducido por la innovación) (Por ter, 1990). De esta forma, más que buscar un elusivo criterio económico objetivo para definir una región, se debería tratar a las regiones como un fenómeno emergente socialmente constituido.

\section{Las complejidades del reescalamiento}

Podemos distinguir cuatro estrategias posibles (de ninguna manera exclusivas ni exhaustivas) que están vinculadas a diferencias en las formas de articulación escalar preferidas. La primera es ampliar o profundizar la división escalar del trabajo dentro de un conjunto integrado de escalas jerarquizadas verticalmente. Esta estrategia típicamente implica la promoción del desarrollo económico (a cualquier escala), aprovechando la dinámica del crecimiento en escalas espaciales progresivamente ascendentes, desde lo local a lo supranacional o global a través de lo regional y nacional. En este contexto, aspectos importantes de la división del trabajo tienden a ser organizados a través de las escalas más que dentro de ellas. Tales estrategias pueden ser promovidas desde arriba y/o emerger desde abajo. Ellas se reflejan en intentos de "posicionar centralmente lugares en 'etapas' de varias escalas espaciales: regionales, na- cionales, internacionales y globales" (Hall \& Hubbard, 1996; Sum, 1999). Tales estrategias también tienden a respetar las fronteras nacionales, pero ellas no son de ninguna manera inconsistentes con las RTF, que son promovidas nacionalmente y donde las autoridades locales de ambos lados de la frontera se orientan hacia sus respectivos centros nacionales en vez de interesarse en consolidar unidades administrativas que buscan autonomía (Nilson, 1997). Esto puede contrastarse con regiones fronterizas en las cuales la frontera es una característica unificadora más que divisoria, por ejemplo donde la frontera funciona para integrar y no dividir (África), y la cual es por tanto planificada como un todo y no como dos partes separadas (Gooneratne \& Mosselman, 1996; Buchanan, 1995).

La segunda opción es construir vínculos horizontales en la misma escala dentro de un conjunto integrado de escalas ver ticalmente articuladas. Tales estrategias son muy amplias, desde la escala local a la triádica. Muchas RTF ilustran esta estrategia, así como alianzas translocales y regiones virtuales. Estas últimas son desarrolladas para vincular localidades no-contiguas con intereses compar tidos o complementarios, tales como la cooperación entre las así llamadas regiones "Cuatro Motores" europeas, que abarcan a Baden-Württemberg, Rhône-Alpes, Lombardía y Cataluña, cada una de las cuales es una ciudad-r egión dinámica asociada con una ciudad no-capital importante. En general, esta estrategia se constr uye sobre intereses e identidades territoriales comunes y busca aprovechar recursos y capacidades de manera conjunta o complementaria. El objetivo es desarrollar una masa crítica por medio de simples economías de aglomeración, o desarrollar una división del trabajo emergente a la misma escala más que a través de escalas. Esta estrategia horizontal puede ser autóctona y/o promovida por cuerpos de estratos superiores o inferiores. De esta forma, las RTF en Eur opa son promovidas por comunidades locales así como por la Unión Europea (UE).

La tercera opción implica la construcción de vínculos transv ersales, por ejemplo saltándose una o más escalas inmediatamente vecinas para buscar una integración más cercana con procesos en varias otras escalas. Esto es especialmente significa- 
tivo donde están implicadas la inversión extranjera directa y la producción para la expor tación, de manera que los vínculos hacia un entorno inmediato o aun hacia la economía nacional pueden resultar mucho más importantes que las conexiones entre las escalas locales y supranacionales. Los triángulos de cr ecimiento en Asia ejemplifican esta estrategia (Parsonage, 1992; Smith, 1997). También las zonas de procesamiento de exportaciones, los puertos libres y las entradas regionales aunque éstas tienden a localizarse dentro de un territorio nacional y orientarse al exterior (ver Chen, 1995, sobre la evolución de las zonas de crecimiento internacionales; Ohmae, 1995, sobre los estados-regiones contemporáneos).

Finalmente, una cuarta opción es buscar un escape para las restricciones locales o escalares localizando las actividades en un espacio sin fronteras o moviéndolas hacia el ciberespacio. Pero esto no obvia la necesidad de algún tipo de localización espacial (islas de ultramar, paraísos financieros, etc.).

Estas opciones pueden ser combinadas para producir estrategias más complejas. Ellas pueden ser aplicadas a un amplio rango de diferentes escalas, y las RTF pertenecen principalmente a las estrategias segunda y tercera. Más generalmente, tales estrategias pueden ser consideradas desde dos puntos de vista: (a) sus agentes principales -agentes económicos privados (firmas, bancos, cámaras de comercio) o agentes públicos (diferentes instancias del gobierno o asociaciones locales o regionales de organismos paraestatales [quangos]); y (b) la naturaleza de la articulación interescalar implicada -vertical (hacia arriba o hacia abajo), lateral (extraversión o introversión), transversal, etc. Mi punto de partida más abajo es la primacía de la escala nacional en el periodo de postguerra, pero esto no debe conducirnos a la conclusión de que la r elativización de la escala implica solamente una internacionalización basada en flujos hacia y desde los espacios económicos nacionales. Pues esta penetración y extraversión es típicamente selectiva, desigual y parcial, y de ninguna forma conecta directamente al conjunto de las economías nacionales.
Consecuentemente, deberíamos examinar las muchas formas diferentes de reescalamiento que están ocurriendo y sus interrelaciones. La lista siguiente no intenta ser exhaustiva, pero sirve para ilustrar el rango de posibilidades que implican a las firmas y estados. Ellas se presentan en orden ascendente desde lo microrregional a lo supranacional a través de los vínculos transversales subnacionales.

Relocalización y re-regionalización, por ejemplo la reemergencia de las economías locales y regionales en las economías nacionales (nuevos distritos industriales, medios innovadores, regiones de aprendizaje, ciudades de entrada, islas mediterráneas como puentes entre Europa y África), o en algunos casos, la descomposición de la economía nacional debida a crisis económicas, guerras civiles, fallas del Estado u otros mecanismos disruptivos. La re-regionalización puede también implicar un incremento en la escala de las regiones (ver Smith, 1988).

Metropolización multicéntrica o polinuclear, por ejemplo una vasta expansión del tamaño o la escala de ciudades principales dentro de las jerarquías urbanas, de forma que ellas se conviertan en grandes regiones metropolitanas o megaurbanas con varios centros.

Interlocalización o interregionalización, por ejemplo el desarrollo de vínculos horizontales entre localidades o regiones contiguas o regiones en la misma escala, pero en estados nacionales diferentes (RTF, alianzas translocales, estados subfederales) que tienen economías o intereses políticos compartidos o complementarios (Hocking, 1999) -vínculos que a menudo se saltan el nivel nacional pero que a veces pueden ser patrocinados por uno o más estados nacionales, así como por autoridades locales, urbanas y regionales, y que se pueden reflejar en el desarrollo de zonas de producción binacional, fronteras internacionales o metrópolis transfronterizas (Albert \& Brock, 1996), y en el desarrollo de corredores internacionales de desarrollo junto a grandes 
vías de transporte (bordes de ríos, costeras, nudos y redes de transporte construidos, etc.). Tales lugares son algunas veces vistos como "glocales", o locaciones globalizadas. En el extremo ellos pueden transformarse en espacios extraterritoriales (centros financieros de ultramar, paraísos financier os, etc.).

- Translocalización or transregionalización, por ejemplo el desarrollo de vínculos horizontales entre localidades y regiones en la misma escala no contiguas, ya sea dentro o a través de los estados nacionales. Boisier (1994) denomina a tales relaciones "regiones virtuales", cuando ellas resultan de construcciones deliberadas de alianzas estratégicas interlocales.

Redes de ciudades globales, por ejemplo la formación de ciudades globales que tienen una fuerte orientación externa hacia otras ciudades globales opuestas al entorno nacional. Esto puede ser visto como un caso especial de transrregionalización, en la medida que las ciudades globales tengan regiones extensas y porque la jerarquía de ciudades globales tiene niveles menor es y nichos especializados, que también forman vínculos transnacionales. Cuando la promoción de tales vínculos resultan en una extraversión de las estrategias de desarrollo urbano que intentan promover la competitividad de una ciudad en la economía global, podemos referirnos a la "glurbanización" (ver Jessop \& Sum, 2000).

Integración mesorregional -la formación de bloques subtríadicos pero supranacionales, como la ASEAN, el MERCOSUR y el CARICOM, los cuales pueden formar bloques para un siguiente nivel de integración o para resistirse a él.

Integración macrorr egional -notablemente la triadización, es decir, la formación de bloques económicos regionales multilaterales estatalmente promovidos que incluyen formalmente varias economías nacionales en América del Norte, Europa y el Noreste de Asia.
Intertriadización-la creciente interpenetración de los bloques de la tríada a medida que las multinacionales residentes en cada bloque forman alianzas estratégicas con socios de otros y se interiorizan los intereses de tales multinacionales dentro de los estados nacionales y de la tríada (ver Poulantzas, 1975).

Transtriadización, el desarrollo de foros y encuentros bilaterales entre diferentes pares de la tríada en la medida que ellos busquen desarrollar y profundizar complementariedades específicas -notablemente a través del foro de Cooperación Económica del Asia Pacífico, la Nueva Agenda Transatlántica y los encuentros Asia-Europa (esto también ha sido discutido bajo la rúbrica de la megarregionalización; ver Hatsuse, 1999).

Globalización propiamente dicha, la introducción y aceptación de normas y estándares globales, el desarrollo de mercados globalmente integrados junto a estrategias orientadas globalmente, firmas sin bases operativas nacionales evidentes y "ubicuidades" (facilidades locacionales uniformemente disponibles).

Estos diferentes procesos y estrategias escalares a menudo se combinan para formar redes y estrategias más complejas, así como enredadas jerarquías de regiones en términos geoeconómicos y geopolíticos. La famosa "Banana Azul"', como una macrroregión gigante, es un ejemplo de ello. Más aun, a medida que emergen nuev as escalas y jerarquías también hay esfuer zos para coor dinarlas. Así, mientras las regiones de la tríada empiezan a adquirir forma e identidad institucional, se desarrollan nuevos foros para coordinar sus relaciones bilaterales. Asimismo, a medida que el regionalismo se desarrolla en la UE, no solamente encontramos un amplio comité de la UE para las regiones, sino también una proliferación de otras asociaciones y vínculos multilaterales entre las regiones. Esto se ve reflejado en la europeización de la política regional y en la regionalización de la polí-

${ }^{3}$ La Banana Azul se curva como una banana desde el Gran Londres a través de los países Benelux, el norte de Francia (saltándose París) y el centro de Alemania, para finalizar en el Norte de Italia. 
tica europea (Balme, 1996). Aún en escalas menor es, las autoridades locales desarrollan asociaciones para promover sus intereses a niveles nacionales, regionales, internacionales y globales. Todo esto produce una creciente complejidad escalar, un alcance creciente para una articulación interescalar deliberada y crecientes problemas para hacer funcionar dichas articulaciones. Temas similares afectan al tiempo y su gobernanza, como se ve en la emergencia de nanotemporalidades a nivel micro y acciones de largo plazo para asegurar la sustentabilidad ambiental a nivel macro. Esto conduce a su vez a crecientes problemas de gobernanza intertemporal.

Tanto en términos geoeconómicos como geopolíticos, los tipos variados de regiones están marcados por grados de hegemonía y jerarquía diferentes y cambiantes, superponiéndose esferas de influencia, componentes nacionales e influencias transnacionales, interdependencias y paquetes de regiones autocontenidas, embrionarias y fallecientes, esferas marginales y áreas de confrontación. Estas complejidades proveen más oportunidades para el reescalamiento, el salto entre escalas y así sucesivamente; ellas también reordenan las jerarquías espaciales y escalar es, produciendo nuevas formas de desarrollo desigual. Esto se refleja no sólo en cambios entre las "economías nacionales", sino también en el auge y caída de regiones, en nuevas formas de división "nor te-sur" y así sucesivamente.

Todo lo anterior apunta al potencial de las alianzas estratégicas entre estados a escalas regionales similares o diferentes (por ejemplo la UE como una organización intergubernamental de estados naciones o como la "E uropa de las regiones") para asegurar las bases de una supervivencia económica y política en respuesta a los imperativos de la competitividad estructural a escala global. Estas alianzas variarán de acuerdo con las posiciones de las economías respectivas en la jerarquía global. Así, mientras que una pequeña economía abier ta (ya sea capitalista, postsocialista o socialista) puede buscar una integración más cercana con el poder económico dominante de su polo de crecimiento triádico inmediato, el poder dominante puede buscar no sólo atraer selectivamente a las economías vecinas dentro de su órbita económica, sino también entrar en alianzas con otros poderes dominantes de la tríada. Una estrategia alternativa para una pequeña economía abierta es buscar nichos de mercado en la economía global (tal vez a través de la promoción de alianzas estratégicas con firmas clave en cada región de la tríada) o formar alianzas regionales con otras economías pequeñas (ya sea que compartan fronteras o no) como la base para incrementar sus capacidades económicas y de presión. Por otra parte, desde que la economía nacional ya no lo es tal, también encontramos regiones subnacionales, ciudades y espacios económicos locales persiguiendo estrategias orientadas a las cambiantes formas de globalización y competitividad internacional.

\section{Regiones transfronterizas}

Las transacciones transfronterizas basadas en proximidades y complementariedades geográficas siempre han existido a niveles regionales y subrregionales, a menos que sean prohibidas por los estados en razón de preocupaciones políticas, de seguridad o económicas (Thant, 1997; sobre el caso europeo ver Gasparini, 1999-2000b; Zago, 1999-2000). Pero las RTF han proliferado siguiendo al declive del proteccionismo, el auge del neoliberalismo, el fin de la Segunda Guerra Fría y los compromisos políticos hacia la integración regional. Las RTF se han convertido en objetos específicos de políticas y no sólo en territorios económicos espontáneos y naturales. En este sentido, representan formas específicas de innovación en relación con el espacio, el lugar y la escala. Ellas implican la producción de nuevos tipos de lugares o espacios para la producción, para el desarrollo de servicios, para el trabajo y para el consumo. Están vinculadas con nuevos métodos de producción de lugares o espacios para crear ventajas específicas de lugares para la producción de bienes y servicios y ofrecer nuevas estructuras regulatorias, infraestructuras, economías de escala, nuev os mercados de trabajo, etc. Pueden implicar la creación de ventajas competitivas aprovechando complementariedades entre fuentes de oferta (como el triángulo de crecimiento de Indonesia-MalasiaSingapur, anteriormente conocido como el triángulo de crecimiento Singapur-Johor-Riau o SIJORI; o los vínculos entre los estados miembros de la UE 
y las economías postsocialistas). Y ellas reconfiguran la jerarquía escalar y modifican la posición de lugares específicos dentro de esta jerarquía.

Hay al menos nueve formas en las cuales han emergido las RTF. Ellas no tienen un peso similar, ya sea histórica o actualmente -por no hablar de casos particulares-, y están típicamente vinculadas con formas muy diferentes de cooperación transfronteriza o interregional. Estas trayectorias alternativas son analizadas de mejor forma junto con las estrategias de articulación interescalar señaladas anteriormente (verticales, horizontales, transversales y virtuales). También debe enfatizarse que estos diferentes patrones no son mutuamente exclusivos. La mayoría de los casos de RTF implican patrones híbridos, ya sea por medio de combinaciones de factores simultáneos o secuenciales (para tales patrones en el caso nórdico, ver Baldersheim y Ståhlberg, 1999). Todo lo que intento lograr aquí es identificar trayectorias analíticamente distintas en el desarrollo inicial, y probablemente, subsiguiente consolidación de las regiones transfronterizas.

Primero, ellas pueden resultar del reciente reforzamiento selectivo de formas oscuras y liminales de organización económica y política que han existido por mucho tiempo en las fronteras de los estados, aun cuando hayan sido desaprobadas por sus respectivos estados nacionales. Estas incluyen actividades económicas "grises" o "negras", el intercambio de "males" o "bienes" (por ejemplo drogas, esclavos, armas, contrabando para evitar aduanas y eximirse de deberes, movimiento de migrantes ilegales). Un ejemplo interesante es el desarrollo de mercados grises (por ejemplo bazares) en las fronteras de la UE con Europa Central, del Este e inclusive más cerca. Tales actividades pueden reflejar una situación periférica compartida de las regiones fronterizas, distantes de los centros. Ellas pueden transformarse en objetos de gobierno o gobernanza para eliminarlas o para transformarlas e integrarlas dentro de estrategias económicas más amplias.

Segundo, ellas pueden implicar el resurgimiento de espacios económicos previamente suprimidos (pero potencialmente viables) con posterioridad al término de la Segunda Guerra Fría, el colapso de la Cortina de Hierro y la apertura de China. Tales regiones fueron algunas veces iniciadas bilateralmente durante la Guerra Fría, como Finlandia-Estonia, Austria-Hungría, GreciaBulgaria (Hettne, 1997). A menudo están vinculadas a recursos compartidos en la frontera (ríos, lagos, costas, bosques); la supervivencia o resurgimiento de antiguas r utas de comercio y/o conexiones heredadas de imperios precoloniales y la existencia de una lengua o etnicidad común. Los ejemplos incluyen la reemergencia parcial del Imperio Austro-Húngaro, el Imperio del Báltico Sueco, la rearticulación de los países Balcánicos que alguna vez pertenecieron al Imperio Otomano, la rearticulación de los estados de Asia Central que alguna una vez integraron grandes formaciones turcoislámicas o iraníes, el desarrollo del Noreste Asiático (China-Rusia-Japón), el surgimiento de la "Gran China”, la gran Subregión de Mekong y el triángulo de crecimiento de SIJORI (el Imperio Johor-Riau). En términos más generales, "la disminución de las tensiones políticas ha motivado a muchos países a abrir sus 'cinturones ocultos', subregiones distantes de los centros comerciales y de intercambio nacionales desarrolladas durante la Guerra Fría, subregiones que ahora pueden vincularse más naturalmente con los mercados mundiales y r egionales emergentes. Este es el caso del Noreste de China, la costa Oeste de Japón, la costa Oeste de Corea, el lejano Este de Rusia y Mongolia" (Jordan \& Khanna, 1995: 445; también sobre la zona de vigilancia del norte de Grecia, ver Labrianidis, 2001). Un factor importante es que algunas regiones fronterizas, que sirvieron como zonas de exclusión defensivas durante la Guerra Fría y que eran consideradas inseguras para inversiones, ahora son vistas como "puentes" articuladores de potenciales socios económicos (Gooneratne \& Mosselman, 1996). Esto se refuerza donde fronteras impuestas desde arriba han dividido regiones "históricas" hasta ahora con identidades propias, y en algunos casos, con agrupamientos étnicos nacionales distintivos.

Tercero, las r egiones fronterizas pueden emerger de (o for talecidas por) derrames de un entorno metropolitano y/o del crecimiento de ciudades complementarias de ambos lados de fronteras compartidas. Muchos ejemplos se encuentran en las 
ciudades gemelas a lo largo de las fronteras entre Canadá y Estados Unidos y entre Estados Unidos y México. Esto también se refleja en los crecientes vínculos Norte-Sur entre regiones canadienses y estadounidenses, aunque aún existe más comercio entre cualquier par de provincias canadienses que entre alguna de ellas y un estado estadounidense.

También hay muchas ciudades fronterizas conectadas cercanamente en Europa (ver Gasparini, 1999-2000a y 1999-2000b). Un ejemplo europeo bien conocido es la región basiliense, que tiene a Basel/Basle como su centro metropolitano. Esta dinámica también puede ser un factor que contribuya al crecimiento de otras RTF, tales como el triángulo SIJORI, que es producto del derrame desde Singapur como una ciudad-Estado en expansión, o el crecimiento económico del Delta del río Pearl, basado en la expansión de Hong Kong después de la aper tura china.

Cuar to, creación de nuevos espacios funcionales económicos o ecológicos donde hay recursos complementarios, problemas económicos o medioambientales comunes, o una situación periférica compartida que denota una necesidad de cooperación en temas tales como el medioambiente o la infraestructura de transporte. Tales RTF a menudo se vinculan con la constitución discursiva de nuevos tipos de territorios o espacios económicos, como triángulos de crecimiento, zonas de procesamiento de exportaciones, medios innovadores, ciudades de entrada, regiones de aprendizaje, biorregiones o ecorregiones (ver Sum, 1999, sobre el triángulo de crecimiento de la G ran China; Lundin \& Söderbaum, 2002, sobre el corredor de Maputo en Africa del Sur; Blatter, 2000, sobre desarrollo sustentable en Cascadia y la Eurorregión Bodenver; Maskell y Törnqvist, 1999, sobre la región transfronteriza de aprendizaje Øresund que une al Copenhague metropolitano con la periferia sur de Suecia). Tales estrategias pueden difundirse a través de una simple imitación, un proselitismo internacional o un patrocinio y financiamiento explícito de instituciones supranacionales. La frontera puede tener una función importante en tales espacios, más que ser visualizada como una barrera para la cooperación. Por ejemplo, Kearny (1991) muestra la uti- lidad de las fronteras para la gestión de mercados de trabajo transnacionales porque ellas pueden servir para mantener la separación espacial del sitio de la compra y del gasto de trabajo de los sitios de su reproducción, en la medida en que los lugares respectivos de producción y reproducción estén en diferentes espacios nacionales (sobre el rol de las patrias durante el régimen del apartheid en Sudáfrica, ver Wolpe, 1988). En este sentido, el éxito de tales estrategias depende de la construcción de nuevos mecanismos de gobernanza para la cooperación transfronteriza sin eliminar, sin embargo, los diferenciales territoriales asociados con la frontera (ver Perkmann, 2000).

Quinto, las RTF pueden ser promovidas por estados nacionales con la esperanza de reestabilizar la escala nacional y permitir a las economías nacionales competir más efectivamente. Esto implicaría la construcción desde arriba de nuevos paradigmas territoriales y la promoción de nuevas escalas de acción territorial, implicando por tanto la disolución y reestructuración de las fronteras. Aquí las RTF actuarían como transmisores y puentes hacia otras escalas nacionales y/o como medios de integrar el espacio económico en bloques regionales y alianzas estratégicas más amplias. Un ejemplo temprano de esto en un contexto europeo fue el trabajo de las comisiones intergubernamentales en Europa. Ellas fueron creadas en los ' $60 \mathrm{y}$ ' 70 a un nivel estatal central para promover el desarrollo de regiones fronterizas, especialmente en el campo de la planificación espacial y del transporte, para el bienestar de las economías nacionales. El interés de esta forma de cooperación económica regional se expandió a fines de los ' 80 y en los ' 90 . Esto probablemente se relaciona muy de cerca con la naturaleza multiescalar, multicénctrica y multiforme de la globalización -incitando a los estados a adaptarse incrementando la competitividad de los sitios locales y regionales de producción, innovación y asignación de capital.

Sexto, y a la inversa, los cuerpos supranacionales pueden promover las RTF para debilitar la escala nacional por medio de un movimiento combinado desde arriba y desde abajo. Esto puede ilustrarse con la estrategia de la "Europa de las regiones". Como indicó el presidente de la Eurorregión: "La UE espera que las regiones for- 
men redes entre las fronteras para permitir contrabalancear el poder de los estados miembros" (J. Chabert, Euroregion Seminar, Brussels, 29.3.94, citado en Taylor, 1995: 77). Esta estrategia implica inventar nuevos modos indirectos y medios de conducir a los niveles menores y a actores no estatales, de forma que ellos se transformen en aliados estratégicos de la Comisión Europea (Tömmel, 1998).

Siete, las RTF pueden ser una reacción al desarrollo desigual vinculado con otros procesos de construcción sub, supra o transnacional de regiones. Las ciudades-Estado de Hamburgo y Bremen han estado promoviendo una nueva Liga Hanseática para contrapesar el dinamismo del polo de crecimiento del sur alrededor de Munich y su propia región transnacional de crecimiento formada por el triángulo Barcelona-Munich-Milán (Hettne, 1997). Asimismo, el crecimiento de la "Banana Azul" gatilló el proyecto del Arco Atlántico para promover a las regiones europeas periféricas que enfrentan el Atlántico; la inversión extranjera directa ha refor zado la primacía de ciudades y regiones centrales de crecimiento en la ASEAN y generó la preocupación de promover triángulos de crecimiento y regiones transfronterizas en áreas más periféricas (Wong, 1994); la inhabilidad de Tokio para dar cuenta de las demandas de desarrollo de localidades en las prefecturas del Mar de Japón han ayudado a gatillar la cooperación transfronteriza en estas zonas; la UE ha promovido la cooperación transfronteriza en las regiones menos favorecidas durante la construcción del Mercado Común. El desarrollo desigual también puede producir una reacción inversa por parte de regiones más fuertes. Murphy (1993) sugiere que una de las razones de la formación de la región de los "Cuatro Motores" fue la preocupación por ser arrastrados hacia abajo por las regiones vecinas pobres.

Ocho, las regiones transfronterizas pueden surgir como parte de proyectos de construcción nacionales en estados territoriales multinacionales. Esto puede reflejar el deseo de fortalecer la autonomía nacional dentro de un sistema federal. Québec es un buen ejemplo de esto: a partir de su incorporación en la CBC como parte de un proyecto de construcción nacional de antigua tradi- ción en las r relaciones exteriores, concluyó muchos acuerdos interregionales en el pasado y persigue actividades de lobby en Estados Unidos (Keating, 1996). Alternativamente, la nación relevante puede compartir fronteras territoriales. El desarrollo de la cooperación transpirinea entre catalanes en España y Francia y el resurgimiento de territorios económicos históricos en países postsocialistas puede ilustrar bien esta situación. Casos más problemáticos, donde existe el derecho a desarrollo de poblaciones transfronterizas, pueden también eventualmente conducir a una cooperación transfronteriza (por ejemplo, el pueblo kurdo).

Nueve, las RTF pueden emerger de iniciativas de construcción de carreras e instituciones a medida que emprendedores políticos aprovechan las oportunidades creadas por la crisis de la escala nacional, el descongelamiento de la Guerra Fría, la disponibilidad de políticas y fondos de la UE, las iniciativas de organizaciones internacionales tales como el B anco de Desarrollo Asiático o el Programa de las Naciones Unidas para el Desarrollo, desr egulación supranacional que permite la re-regulación regional, el desarrollo de nuevas infraestructuras y logísticas (ver especialmente Perkmann, 2000). Tales casos de simple cálculo económico pueden reflejarse en coaliciones para la obtención de fondos (más que coaliciones para el crecimiento) y en simples respuestas a ventanas de oportunidades políticas para iniciativas políticas.

A pesar de su nueva importancia económica, las RTF carecen tanto de límites sólidos demarcados por fronteras nacionales, así como de muchas de las condiciones institucionales macroeconómicas consideradas a menudo esenciales para un crecimiento económico estable. Es en este contexto que las redes interpersonales y la negociación interorganizacional resultan cruciales para unir la división público-privado entre las fronteras y para asegurar la cooperación de los denominados "actores clave" surgidos de diferentes sistemas funcionales. Más aun, la ausencia de un Estado con soberanía sobre una RTF puede no ser un aspecto en contra, como a veces creen muchos de los directiv os de los estados nacionales. Pues si ni las puras fuerzas del mercado ni el comando desde arriba por parte de un centro político pueden garantizar la competitividad sistémica o estructural de un espa- 
cio económico, puede haber lugar para una búsqueda más cooperativa para proveer de una estructura estable de acción económica, a través de otros medios más flexibles y no jerárquicos.

Otra forma de avanzar en estos desarrollos es a través del esquema de Messner (1998) sobre los diferentes niveles de organización relevantes para la búsqueda de la competitividad sistémica. Él distingue cuatro aspectos o niveles de competitividad: micro, meso, macro y meta. En una era de globalización, la competitividad aún depende tanto de ventajas genéricas así como de ventajas específicas de lugar, y esto será particularmente cierto para las fuentes a nivel meta y micro. Entonces, podríamos sugerir que en el caso de las RTF, los niveles micro y meta serán más significativos que el nivel macro, y que el nivel meso será importante pero modificado, a la luz de la naturaleza transfronteriza de la economía. A su vez, estas son ár eas particularmente apropiadas de intervención para mecanismos de gobernanza que no descansan ni en las fuer zas anárquicas del mercado ni en la planificación desde arriba, sino que en diversas formas, tales como redes y asociaciones públicoprivadas, acuerdos sociales entre diversos interesados, etc. (sobre gobernanza, ver Jessop, 1998). Hay un alcance considerable aquí para la metagobernanza (por ejemplo, el Consejo Europeo provee modelos de reglas organizacionales para los acuerdos sobre RTF según la Convención sobre Cooperación Transfronteriza).

\section{Regiones transfronterizas y reestructuración del Estado}

Estas complejas formas de reescalamiento, que como se sugirió, van desde la relocalización hasta la globalización, se asocian típicamente con tres tendencias principales en el Estado y la política. Éstas abarcan la desnacionalización del Estado territorial, la creciente importancia de las asociaciones público-privadas y de redes y un énfasis creciente sobre vínculos funcionales y responsabilidades conjuntas sin considerar la posición dentro de jerarquías escalares y la internacionalización de los regímenes de políticas.

Cada una de estas tendencias es discernible en el desarrollo de las regiones tranfronterizas, pues ellas ilustran la desnacionalización pero aún son territoriales, están basadas típicamente en una gobernanza multinivel y reflejan las preferencias internacionales de políticas. A la inversa, el desarrollo de las RTF tiende tanto a refor zar estas tendencias y a provocar contratendencias en el nivel nacional.

La primera tendencia es la desnacionalización de la estatalidad. Esto se refleja empíricamente en el "vaciamiento" de los aparatos del Estado-nación, donde antiguas y nuevas capacidades estatales están siendo territorial y funcionalmente reorganizadas a niveles subnacionales, nacionales, supranacionales y translocales. Un aspecto es la pérdida parcial de soberanía jurídica por parte de los estados nacionales en ciertos aspectos, a medida que ellos transfieren poderes de toma de decisiones hacia arriba hacia cuerpos supranacionales o suscriben tratados con obligaciones más o menos asimétricas. En ambos casos, las nuevas reglas y decisiones se convierten en ataduras para los estados nacionales. El primer caso es más evidente en la UE, y el segundo caracteriza al NAFTA y otros bloques regionales organizados intergubernamentalmente y cuerpos internacionales, tales como la Organización Mundial de Comercio. Otro aspecto es la descentralización de la autoridad hacia niveles subordinados de organización territorial y/o el desarrollo de toma de decisiones transnacional pero interlocal. Las RTF son significativas a este respecto, tanto por el fortalecimiento de los estados regionales o locales en el desarrollo económico, así como por el desarrollo de vínculos transnacionales entre autoridades regionales y locales, que implica lo que se ha denominado a veces la "paradiplomacia" (Dommergues, 1992) o la política "interméstica" (Duchacek, 1986). De esta forma, la emergencia de las RTF parece desafiar la supervivencia del Estado nacional, aunque de otra forma que en el reescalamiento supranacional.

Sin embargo, contradiciendo esta tendencia hacia la desnacionalización del Estado, se encuentra la continua supervivencia en muchas sociedades del Estado nacional como el principal factor de cohesión. Por todas partes, sin embargo, los estados nacionales están en crisis o ya se han descompuesto. Pero donde el Estado nacional como 
forma sobrevive institucionalmente, también retiene sus funciones políticas generales cruciales, aun cuando fuerzas técnico-económicas, políticas e ideológicas específicas se transfieran hacia otras partes. Pues las formas supranacionales de organización política son de carácter típicamente intergubernamental, y el Estado nacional está implicado en la transferencia de poder hacia arriba y legitima esta transferencia. Como estados soberanos, ellos también retienen el poder formal para recentralizar el poder delegado hacia abajo y para regular los contactos y acuerdos translocales. Algunos estados son más poderosos y efectivos que otros respecto de la articulación interescalar, siendo el imperial Estados Unidos el actor estatal más poderoso en este sentido. Por otra parte, en muchas RTF, las unidades administrativas locales o regionales implicadas en los diferentes lados de la(s) frontera(s) actúan como mecanismos de transmisión de la política nacional, y están más orientadas hacia sus respectivos estados nacionales que a forjar relaciones políticas que debiliten la soberanía jurídica nacional. En este sentido, los estados nacionales actúan como "pivotes" entre las agencias internacionales y las actividades subnacionales, porque ellos proveen legitimidad como voz exclusiva de una población territorialmente delimitada (Hirst, 1997). Sin embargo, su capacidad para realizar lo anterior continuará dependiendo de su capacidad para asegurar la cohesión social.

Segundo, hay una tendencia general hacia la desestatización de los regímenes políticos. Esto se refleja empíricamente en el giro desde el gobierno a la gobernanza en varias escalas territoriales y a través de varios dominios funcionales. Los gobiernos siempre han descansado en otras agencias para ayudarse a realizar objetivos estatales, pero ahora hay un reordenamiento político significativo en la relación gobierno y gobernanza. Aunque esta tendencia implica típicamente una pérdida de autonomía decisional y operacional de los aparatos del Estado (a cualquier nivel), también puede fortalecer sus capacidades para proyectar el poder del Estado y alcanzar objetivos colectivos por medio de la movilización de recursos de conocimiento y poder de influyentes socios no gubernamentales e interesados. Esta segunda tendencia ocurre no sólo a nivel internacional o nacional, sino que tam- bién es evidente en la reestructuración de la gobernanza regional, local y transfronteriza. De hecho, las RTF a menudo se caracterizan por complejas formas de gobernanza multinivel, que implican a un amplio rango de socios y otros interesados reclutados de distintos dominios funcionales y escalas de organización territorial. También significa que los propios estados se están transformando en socios, facilitadores y árbitros en consor cios público-privados, coaliciones de crecimiento, etc., más que en los impulsores primarios en muchas áreas de políticas.

Contradiciendo este giro desde el gobierno a la gobernanza hay una tendencia a que el gobierno adquiera un rol más fuer te en la metagobernanza. Esta última se refiere al rol de las autoridades políticas (a escala nacional u otras) en la organización directa o indirecta de la autoorganización de sociedades interorganizacionales, redes y relaciones intersistémicas. Esto se necesita para limitar la proliferación de mecanismos de gobernanza. P ues esta última puede conducir a una creciente complejidad no estructurada y a la emergencia de fallas de gobernanza tan significativas como las fallas del mercado o del Estado, y con escapatorias crecientes para aprovechadores inescr upulosos y con brechas crecientes en las redes de seguridad para los menos afortunados (sobre la gobernanza y metagobernanza, ver Jessop, 1998).

Hay peligros claros de esto en las RTF sin mecanismos de gobernanza bien diseñados. Así, al expandir su rol en la metagobernanza, los estados asumen la responsabilidad para el diseño constitucional y otras medidas para asegurar una compatibilidad relativa de diferentes mecanismos de gobernanza. Esto se refleja en el Consejo de la Convención Europea sobre Cooperación transfronteriza $y$ en las regulaciones de la UE que gobiernan la naturaleza de los acuerdos y consultas sobre asociaciones necesarias para recibir la aprobación y el financiamiento de la UE. Adicionalmente, los estados (espacialmente a nivel nacional) deben responder en última instancia a las repercusiones políticas de la gobernanza así como a las fallas de mercado. Si la metagobernanza funciona o no, es otro problema. 
Tercero, hay una tendencia general hacia la internacionalización de los regímenes de políticas. Esto se refiere a la creciente significación estratégica del contexto internacional sobre la acción estatal doméstica y la extensión de esta última a un amplio rango de procesos y factores extraterritoriales o transnacionales. Esto tiende a hacer desapar ecer la distinción entre la política doméstica y extranjera y a ampliar las bases territoriales de actor es que estén directamente involucrados en la toma de decisiones, y/o cuyas opiniones y probables reacciones sean tomadas en cuenta. Esta tendencia no está de ninguna manera confinada a los estados nacionales. También es aplicable a bloques supranacionales (tales como la UE), a estados locales o regionales, y con particular claridad, a las nuevas RTF emergentes. Todo esto también necesita tener en cuenta el cambiante contexto internacional de sus actividades económicas. Inter alia, esto se refleja en los intentos de combinar el desarrollo económico endógeno con inversiones externas así como involucrarse en actividades de promoción de exportaciones y/o de sustitución de importaciones en una economía internacional continuamente cambiante.

Aquí también hay una contratendencia, no obstante con dos aspectos. Por una parte, hay una creciente interiorización de las restricciones internacionales, como su integración en el paradigma de políticas y modelos cognitivos del mundo sostenidos por varios tomadores de decisiones domésticos. Por otra parte, hay un esfuerzo más intenso, especialmente por parte de los estados más poderosos, para influenciar la forma y contenido de los regímenes internacionales. El peso relativo de estas tendencias y sus contratendencias variarán claramente según el lugar de un espacio económico y político dado, dentro del orden económico y político más general.

\section{Conclusiones}

Hay muchas más RTF, y aun más proyectos de RTF que las que existen como ejemplos exitosos. La viabilidad de los proyectos depende en parte, por cier to, de la capacidad de narrativas y otras formas discursivas para obtener apoyo para los proyectos, para permitir a las fuerzas apropia- das prever un futuro alternativo y para identificar la trayectoria que debe ser seguida para alcanzarla. Per también se requieren condiciones extradiscursivas importantes para lograr estrategias plausibles. Estas condiciones extra-discursivas tienen su raíz en la materialidad de órdenes institucionales diferenciados (sistemas funcionales) y sus complejas formas de interdependencia a lo largo de varios horizontes de acción espaciales y temporales. Esto, a su vez, depende del deseo político y de las capacidades de liderazgo, incluyendo las capacidades para la metagobernanza.

Por lo tanto, ninguna estrategia regional o subregional tiene la probabilidad de convertirse en predominante. En vez de eso, habrá un gran número de iniciativas estratégicas a pequeña escala que interactuarán con un número aun mayor de desarrollos más espontáneos con raíces en los intercambios de mercado, la inversión extranjera directa, etc. Las estrategias económicas regionales tienen mayor probabilidad de desarrollarse a través de una colaboración descentralizada e iniciativas conjuntas, en las cuales las regiones fronterizas y/o las afinidades económicas jugarán un rol mediador clave. La enorme multiplicidad de estas iniciativas puede ser vista a partir de una lista parcial de programas para la renovación económica provista por Neumann (1994) para la región del Báltico Norte. Estas incluyen una nueva Liga Hanseática, la Región del Mar Báltico, el Mare Balticum, la Región Euro-Báltica, el Espacio Político Scann-Báltico, un Ostverraum, un severobaltiyskiy poyas (cinturón nor-báltico que incluye los estados nórdicos y las cuatro entidades políticas rusas de Murmansk, San Petersburgo, Karelia, y Kaliningrad $)^{4}$, y una región Barents centrada en Noruega y extendiéndose hacia los mares Ártico y del Norte. Una profusión similar de proyectos existe para otras regiones en Europa y Eurasia.

Si bien no todos estos proyectos puede ser exitosos, muchos probablemente atraerán apoyo político y recursos económicos. De esta forma, eventuales configuraciones regionales serán fenómenos complejos, enredados y evolutivos (más que plani-

${ }^{4}$ Nótese que la propuesta del cinturón del Báltico Norte excluye tanto a Polonia como a los Länder alemanes que bordean el Báltico. 
ficados completamente). Si emergiera una escala principal probablemente será a nivel de la tríada. Esto es particularmente evidente en la UE (especialmente a medida que continúe ampliando y profundizando su rol en la estructuración del espacio económico europeo) y en el NAFTA (con el abrumador dominio de Estados Unidos), pero también existe una creciente división regional del trabajo en el Este Asiático, y China se está transformando en un actor significativo en este aspecto.

\section{Referencias bibliográficas}

Albert, M. \& L. Brock (1996) "De-bordering the State: new spaces in international relations". New Political Science, 35: 69-107.

Baldersheim, H. \& K. Ståhlberg (eds.) (1999). Nordic Region-Building in a European Perspective. Aldershot: Ashgate.

Balme, R. (ed.) (1996). Les politiques du néorégionalisme. Action collective régionale et globalisation. Paris: Economica.

Blatter, J. (2000). "Emerging cross-border regions as a step towards sustainable development? Experiences and considerations from examples in Europe and North America". International Journal of Economic Development, 2, 3: $402-$ 439.

(2001). "Debordering the world of states. Towards a multi-level system in Europe and a multi-polity system in North America? Insights from border regions". European Journal of International Relations, 7, 2: 175210.

Boisier, S. (1994). "Regionalization processes: past crises and curr ent options". CEPAL Review, 52: 177-89.

Brenner, N. (1998). "Global cities, glocal states: global city formation and State territorial restructuring in contemporary Europe". Review of International Political Economy, 5, 1: 1-38.

Brenner, N., M. Jones, B. Jessop y G. MacLeod, Gordon (eds.) (2003). Statelspace: a reader. Oxford: Blackwell.

Buchanan, R. (1995). "Border crossings: NAF$\mathrm{TA}$, regulatory restructuring, and the politics of place”. Indiana Journal of Global Legal Studies, 2, 2.
Chen, X. (1995). "The evolution of free economic zones and the recent development of crossnational growth zones". International Journal of Urban and Regional Research 19, 4: 593621.

Collinge, C. (1996). Spatial articulation of the State: reworking social relations and social regulation theory. Birmingham: Centre for Urban and Regional Studies.

Dommergues, P. (1992). "The strategies for international and interregional cooperation". Ekistics, 352-353: 7-12.

Duchachek, I. D. (1986). The territorial dimension of politics. Boulder: Westview.

Gasparini, A. (1999-2000a). "European border towns as laboratories of differentiated integration”. ISIG Magazine (Istituto di Sociologia Internazionale di Gorizia), 8, 4 y 9, 1: 2-3.

(1999-2000b). "Situations, conditions, styles of life and government in border towns". ISIG Magazine (Istituto di Sociologia Internazionale di Gorizia), 8, 4 y 9, 1: 4-9.

Gooneratne, W. \& E. E. Mosselman (1996). "Planning across the borders': border regions in Eastern and Southern Africa”. Regional Development Dialogue, 17, 2: 136-54.

Hall, T. \& P. Hubbard (1996). "The entrepreneurial city: new urban politics, new urban geographies?" Progress In Human Geography, 20, 2: 153-74.

Hansen, N. (1986). "Border r egion, development and co-operation”. Martínez, O. J. (ed.), Across Boundaries. El Paso: Western Texas Press.

Hatsuse, R. (1999). "Regionalisms in East Asia and the Asia-Pacific". Yamamoto, Y. (ed.), Globalism, regionalism and nationalism: Asia in searh of its role in the twenty-first century. Oxford: Blackwell.

Herzog, L. A. (1991). "Cross-national urban structure in the era of global cities: the USMexico transfrontier metropolis". Urban Studies, 28, 4: 519-33.

Hettne, B. (1997). "Europe in a world of regions". Falk, R. \& T. Szentes (eds.), A new Europe in the changing global system. Tokyo: United Nations University Press. 
Hirst, P. Q. (1997). “The global market and the possibilities of governance". Paper for Conference on Globalisation -Critical Perspectives. University of Birmingham, 1416 March.

Hocking, B. (1999). "Patrolling the 'Frontier': globalization, localization and the 'actorness' of non-central governments". Regional and Federal Studies, 19, 1: 17-39.

Jenson, J. (1995). "Mapping, naming and remembering: globalization at the end of the twentieth century". Review of International Political Economy, 2, 1: 91-116.

Jessop, B. (1998). "The rise of governance and the risks of failure". International Social Science Journal, 155: 29-46.

(1999). "Reflections on the (il)logics of globalization”. Olds, K., P. Dicken, P. F. Kelly, L. Kong y H. Wai-Cheung Yeung (eds.), Globalization and the Asia Pacific: contested territories. London: Routledge. (2002). The future of the capitalist state. Cambridge: Polity.

Jessop, B. \& Ng.-L. Sum (2000). “An entrepreneurial city in action: Hong Kong's emerging strategies in and for (inter-)urban competition”. Urban Studies, 37, 12: 2290 315.

Jordan, A. \& J. J. Khanna (1995). “Economic interdependence and challenges to the nationState: the emergence of natural economic territories in the Asia-Pacific". Journal of International Affairs, 48, 2: 433-62.

Kearney, M. (1991). "Borders and boundaries of State and self at the end of empire". Journal of Historical Sociology, 4, 1: 52-74.

Keating, M. (1996). "Les provinces canadiennes dans la concurrence Inter-régionale nordaméricaine". Balme, R. (ed.), Les politiques du néo-régionalisme. Action collective régionale et globalisation. Paris: Economica.

Krätke, S. (2001). "Cross-border co-operation in the German-Polish border area". van Geenhuizen, M. \& R. Ratti (eds.), Gaining advantage from open borders. An active space approach to regional development. Aldershot: Ashgate.

Labrianidis, L. (2001). “Internal Frontiers' as a hindrance to development". European
Planning Studies, 9, 1: 29-46.

Leresche, J.-P. \& G. Saez (2002). "Regional identities and political border regimes". Perkmann, M. \& N.-L. Sum (eds.), Globalization, regionalization, and cross-border regions. Basingstoke: Palgrave.

Lundin, I. B. \& F. Söderbaum (2002). "Microregionalism and the construction of development in South Africa: the case of the Maputo Corridor”. Perkmann, M. \& N.-L. Sum (eds.), Globalization, regionalization, and cross-border regions. Basingstoke: Palgrave.

Maskell, P. \& G. Törnqvist (1999). Building a cross-border learning region. Emergence of the North European Øresund region. Copenhagen: Copenhagen Business School Press.

Messner, D. (1998). The network society: economic development and international competitiveness as problems of social governance. London: Cass.

Murphy, A. B. (1993). "emerging regional linkages within the European Community: Challenging the dominance of the State". Tijdschrift voor Economische en Sociale Geografie, 84, 2: 103-18.

Neumann, I. B. (1994). "A region-building approach to Northern Europe". Review of International Studies, 20, 1: 53-74.

Nilson, H. R. (1997). "Nordic regionalization: on how transborder regions work and why they don't work". Cooperation and Conflict, 32, 4: 399-425.

Ohmae, K. (1995). The end of the nation State: the rise of regional economies. New York: Free Press.

Paasi, A. (2001). "Europe as a social process and discourse: considerations of place, boundaries and identity". European Urban and Regional Studies, 8, 1: 7-28.

Parsonage, J. (1992). "Southeast Asia's 'Growth Triangle': a subregional response to global transformation". International Journal of Urban and Regional Research, 16, 3: 307-17.

Perkmann, M. (2000). Euroregions: strategies of institution-building in the new European polity. Lancaster: Lancaster University, Ph.D. thesis.

Porter, M. E. (1990). The competitive advantage of nations. Basingstoke: Macmillan.

Poulantzas, N. (1975). Classes in contemporary capitalism. London: New Left Books. 
Scott, J. W. (2002). "On the political economy of transboundary co-operation in North American 'maquiladoras' and regional development issues on the US-Mexican border”. Perkmann, M. \& N.-L. Sum (eds.), Globalization, regionalization, and cross-border regions. Basingstoke: Palgrave.

Smith, M. P. (2000). Transnational urbanism: locating globalization. Oxford: Blackwell.

Smith, N. (1988). "The region is dead! Long live the region!”. Political Geography Quarterly, 7, 2: 141-52.

Smith, S. L. D. (1997). “The Indonesia-MalaysiaSingapore growth triangle: a political and economic equation". Australian Journal of Intemational Affairs, 51, 3: 369-82.

Sparkes, M. (2002). "Not a State, but more than a state of mind: cascading Cascadias and the geo-economics of cross-border regionalism". Perkmann, M. \& N.-L. Sum (eds.), Globalization, regionalization, and cross-border regions. Basingstoke: Palgrave.

Swyngedouw, E. A. (1997). "Neither global nor local. 'Glocalization' and the politics of scale”. Cox, K. (ed.), Spaces of globalization. Reasserting the power of the local. New York: Guilford.
Sum, N.-L. (1999). "Rear ticulating spatial scale and temporal horizons of trans-border space". Olds, K., P. Dicken, P. F. Kelly, L. Kong y H. Wai-Cheung Yeung (eds.), Globalization and the Asia Pacific: contested territories. London: Routledge.

Taylor, K. (1995). "European Union: the challenge for local and regional government". Political Quartenly, 66, 1: 74-83.

Thant, M. (1997). "Economic cooperation in the Greater Mekong subregion”. Asia-Pacific Economic Literature, 11, 2: 40-57.

Tömmel, I. (1998). "Transformation of governance: the European Commission's strategy for creating a 'Eur ope of the regions'”. Regional and Federal Studies, 8, 2: 52-80.

Wolpe, H. (1988). Race, class and the apartheid State. London: James Currey.

Wong, S. Y. (1994). "Globalization and regionalization: the shaping of new economic regions in Asia and the Pacific". Regional Development Dialogue, 15, 1: 3-22.

Zago, M. (1999-2000). "The State of cooperation between border towns". ISIG Magazine (Istituto di Sociologia Internazionale di Gorizia), 8, 4 y 9, 1: 10-20. 